\title{
THE VULNERABILITY OF THE POSTERIOR INTEROSSEOUS NERVE OF THE FOREARM
}

\author{
A Case Report and an Anatomical Study
}

\author{
Norman Capener, Exeter, England
}

In this paper I report a case of paresis of the posterior interosseous nerve due to a tumour, together with a study of anatomical relations which hitherto have not been well recognised. These I think have some influence in certain cases of "tennis elbow." These anatomical studies are based upon observations made during operations supported by wider dissections in cadavers*.

Case report-Compression of posterior interosseous nerve by a lipoma. A company director aged fifty-eight attended with weakness of the left hand which developed gradually over two weeks, and with a swelling over the antero-lateral aspect of the forearm just below the elbow. He said that, two months before, he was moving a load of bricks in a wheelbarrow which suddenly overturned to the left, forcibly twisting the left forearm. He had no particular pain or swelling at the time, but he rapidly lost all power in the long extensors of the fingers and thumb. There was no sensory disturbance. In view of the reported injury it seemed possible that he had a haematoma with pressure upon the posterior interosseous nerve.

At operation, the lateral aspect of the upper part of the forearm was exposed through a vertical incision from the lateral epicondyle immediately in front of the interval between the extensor carpi radialis brevis and the extensor digitorum communis. This is the site of a deep septum which will be described later. Beneath the carpal extensor there was a bulging mass seven centimetres in diameter (Fig. 1). It was lying deep to the aponeurotic and muscular fibres of the supinator muscle which were stretched across it. These fibres were split vertically to reveal a large lipoma. It lay superficially to the posterior interosseous nerve. A prolongation of the lipoma extended upwards over the front of the head of the radius and was much constricted at a point which corresponded to the upper oblique tendinous fibres of the supinator muscle. After this had been freed and the tumour removed, the nerve was dissected and showed a well-marked area of bruising and discoloration at the point where it had been constricted (Fig. 2). Five years later the patient's forearm had completely recovered.

\section{DISCUSSION}

This patient's condition had particular interest for me because I had been studying the clinical and anatomical features of "tennis elbow." One was aware of the many lesions which had been described in this disorder, which clearly is due to repetitive stress in occupations requiring forceful action involving pronation, supination and extension of the wrist. In operations upon the attachments of the extensor muscles to the epicondyle I had seen no evidence of epicondylitis, torn muscle fibres, myositis, bursitis nor evidence of impingement of synovial fringes in the radio-humeral joint.

The need for operation is rare because most patients with this form of occupational lesion get well by simpler treatment or by no treatment at all. In a few chronic cases (ten elbows) I have explored the posterior interosseous nerve because I have found that, apart from the typical pain and tenderness at the lateral epicondyle of the humerus and lateral joint line, there has been extreme discomfort on pressure over the line of the nerve in front

\footnotetext{
* Since this paper was submitted, a further dissection of the posterior interosseous nerve has been done by Dr D. H. Thompsett, Prosector at the Royal College of Surgeons of England, which confirms the details reported here and contains additional information to be published elsewhere.
} 


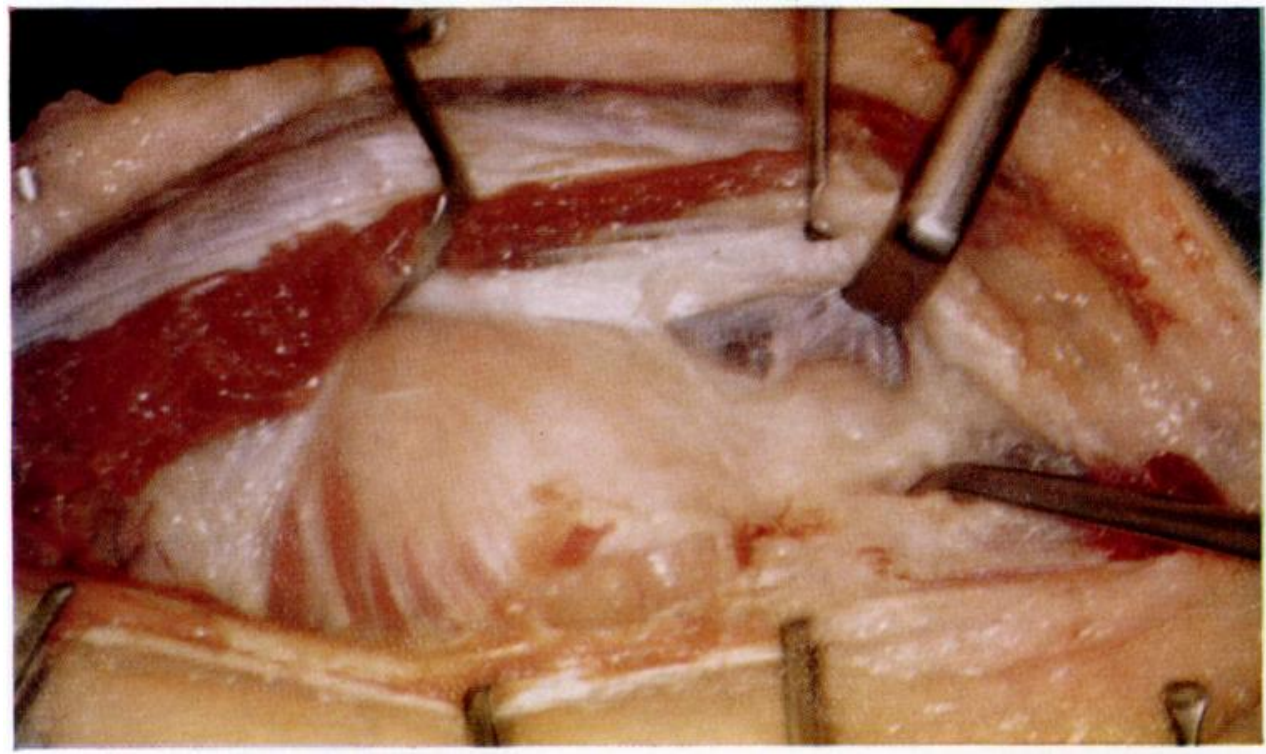

Fig. 1

Left elbow-lateral view. Operative dissection of interval in front of the intermuscular septum exposing the lipoma which is covered by thinned out fibres of supinator muscle. The upward extension of the tumour is seen to the right of the picture.

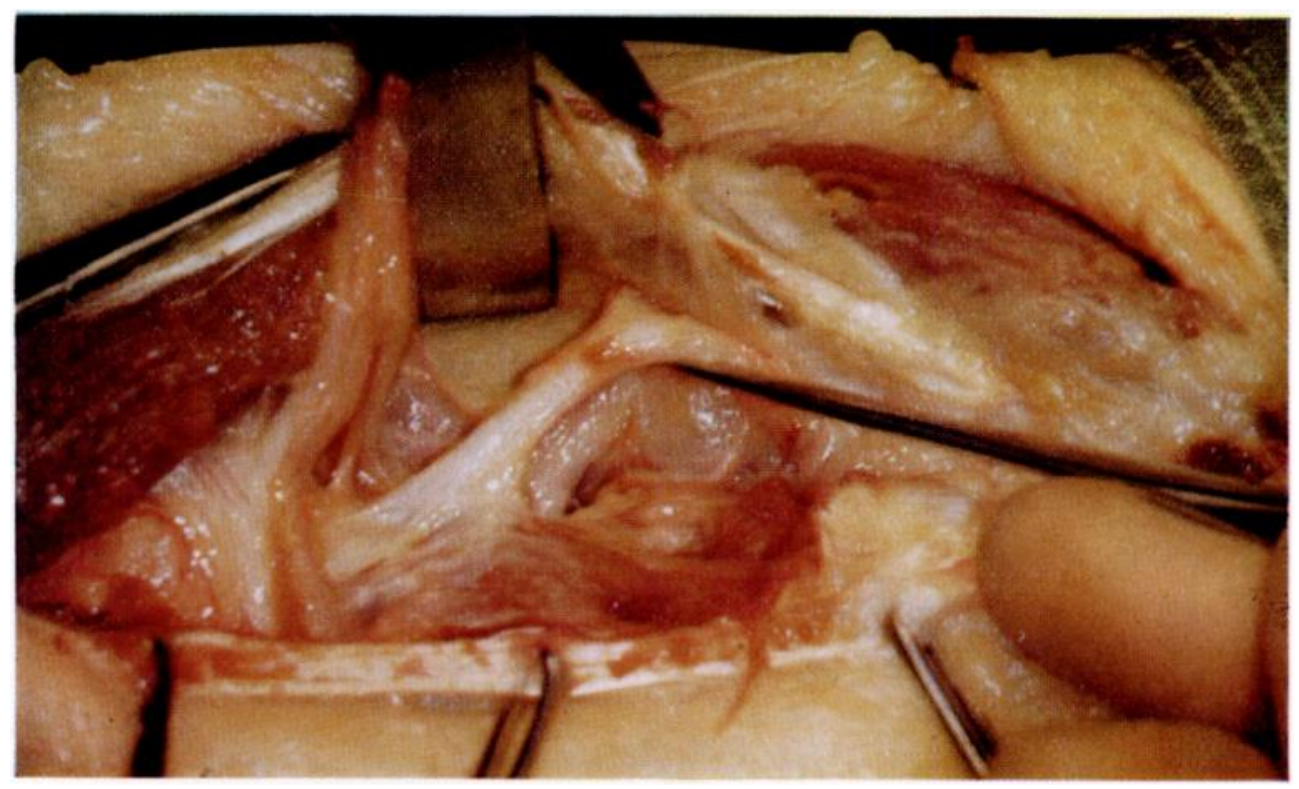

FIG. 2

After removal of the tumour and its upward extension; the area of constriction in the posterior interosseous nerve is seen. 


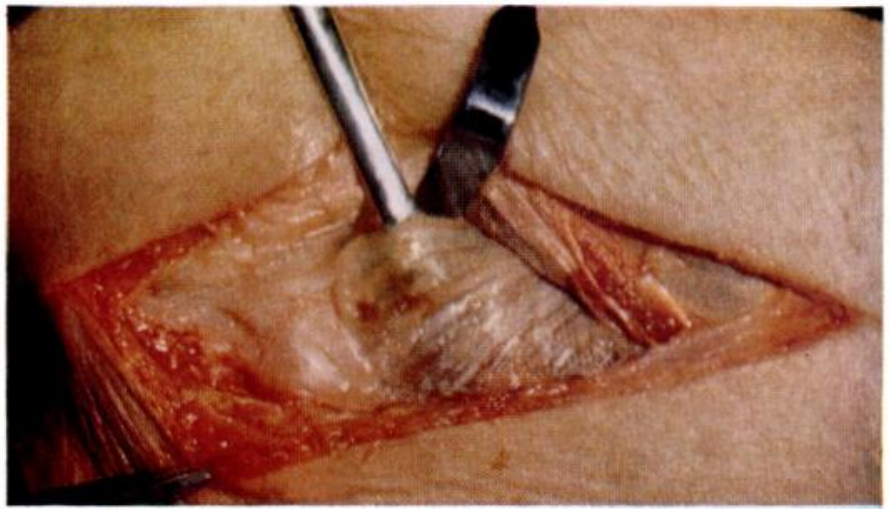

FIG. 3

Right elbow-postero-lateral view. Operative dissection of interval behind the intermuscular septum exposing the aponeurotic covering of the supinator.

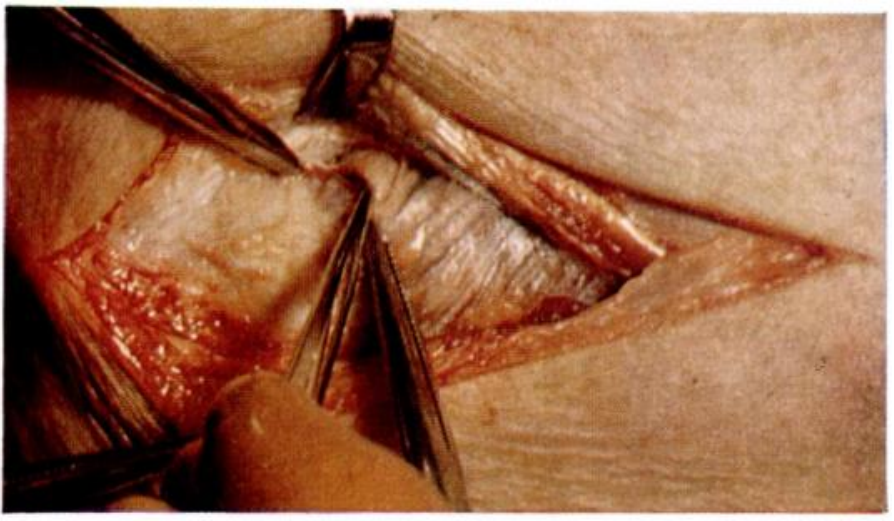

Fig. 4

Exposure of the posterior interosseous nerve above the sharp aponeurotic edge.

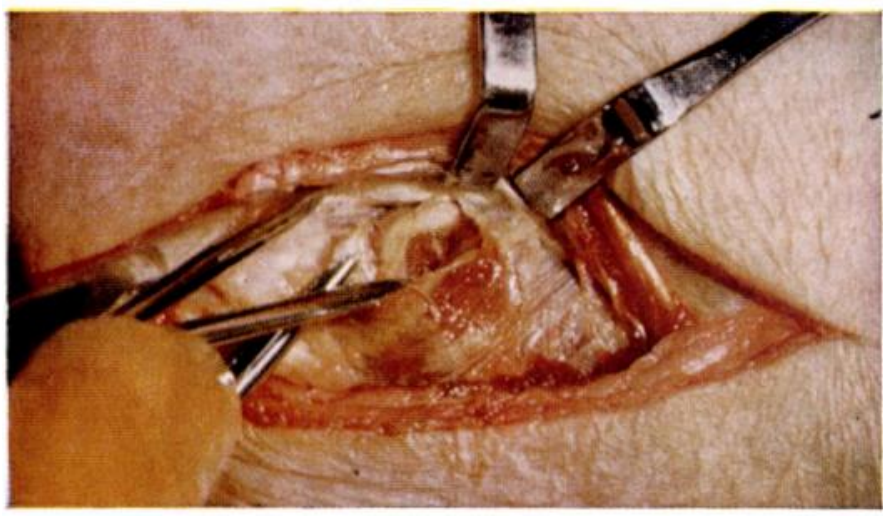

Fig. 5

Division of aponeurotic and fleshy muscular fibres to decompress the nerve. (Note the bulge above the area of constriction.) 
of the neck of the radius. Quite often in these patients pain is also referred to the back of the carpus and there is, as is well known, weakness of the extensor muscles of the wrist and digits. The possibility of a local neurological lesion seemed worth investigation. In at any rate some cases I think there is, but the number of patients in which the nerve has been explored and decompressed is too small for statistical review. The results not less than one year afterwards are not outstandingly brilliant, for in the ten elbows, five were completely relieved of symptoms, three had marked improvement, one was no better and one patient could not be traced.

Decompression of the nerve-An incision is made from the tip of the lateral epicondyle with the forearm in mid pronation-supination and the elbow flexed to nearly a right angle. It is in a vertical line for ten centimetres, following the interval between the extensor carpi radialis brevis and the extensor digitorum communis. This interval is usually seen as a groove in the skin which corresponds to a dense aponeurotic septum between these two muscles. The fibres of the extensor digitorum are separated from this septum, mostly by blunt dissection and the muscle is retracted posteriorly. The upper attachment of the septum is divided from the lateral epicondyle and the structure is retracted forwards to expose the oblique fibres of the supinator muscle which are covered by a thin but definite aponeurotic sheet. By blunt dissection this is followed upwards until a sharp thickened edge is revealed (Fig. 3). Deep to this edge the posterior interosseous nerve and its accompanying vessels pass into a cleft which is completed above by another aponeurotic sheet that covers the transverse fibres of the supinator deep to the nerve (Fig. 4). At operation it can clearly be demonstrated how the interval narrows when the radius is pronated passively or in active supination. During supination the nerve also comes to lie deep to the intermuscular septum. To free the nerve it is only necessary to split the aponeurotic and fleshy muscular fibres covering it (Fig. 5).

If, as I believe, there are some cases of typical "tennis elbow" in which there is a disturbance of the posterior interosseous nerve, there must be neuritis possibly of central origin (such as cervical spondylosis). But that is another story.

\section{SUMMARY}

1. The posterior interosseous nerve of the forearm is vulnerable to constriction in an aponeurotic cleft in the supinator muscle.

2. A case is presented of paresis of the nerve by an intramuscular lipoma which extended into this cleft.

3. A discussion is given of the possible relation of this feature to certain cases of occupational stress-" tennis elbow."

4. An approach is described for decompressing the nerve in the rare cases which do not respond to conservative treatment.

\section{REFERENCE}

CAPEner, N. (1960): Report of the Annual Meeting of the British Medical Association, Torquay. British Medical Journal, ii, 130.

VOL. 48 B, NO. 4, NOVEMBER 1966 